Revue critique de fixxion française contemporaine

22 | 2021

Figures du mensonge et de la mauvaise foi dans le roman contemporain

\title{
Mensonges à grande échelle dans la trilogie d'Antoine Bello. Re(con)figurations d'un imaginaire complotiste transséculaire
}

Chloé Chaudet

\section{(2) OpenEdition}

Édition électronique

URL : https://journals.openedition.org/fixxion/317

DOI : $10.4000 /$ fixxion.317

ISSN : 2295-9106

Éditeur

Ghent University

Référence électronique

Chloé Chaudet, « Mensonges à grande échelle dans la trilogie d'Antoine Bello. Re(con)figurations d'un imaginaire complotiste transséculaire », Revue critique de fixxion française contemporaine [En ligne], 22 | 2021, mis en ligne le 15 juin 2021, consulté le 17 février 2022. URL : http://journals.openedition.org/ fixxion/317 ; DOl : https://doi.org/10.4000/fixxion.317

Ce document a été généré automatiquement le 17 février 2022.

\section{(c) (1) (9)}

Les contenus de la Revue critique de fixxion française contemporaine sont mis à disposition selon les termes de la licence Creative Commons Attribution - Pas d'Utilisation Commerciale - Pas de Modification 4.0 International. 


\title{
Mensonges à grande échelle dans la trilogie d'Antoine Bello. $\operatorname{Re}($ con)figurations d'un imaginaire complotiste transséculaire
}

\author{
Chloé Chaudet
}

Comme l'écrivit Michelet, "l'Encyclopédie fut bien plus qu'un livre, la conspiration victorieuse de l'esprit humain".

Antoine Bello, Les éclaireurs (2009)

1 La série de romans Les falsificateurs (2007) ${ }^{1}$, Les éclaireurs $(2009)^{2}$ et Les producteurs (2015) a valu à Antoine Bello un succès rapide. Si certains ont pu admirer la popularité soudaine de l'écrivain franco-états-unien ${ }^{4}$, force est de constater que les figures du mensonge qui parcourent sa trilogie ne sont pas tout à fait nouvelles. Le "Consortium de Falsification du Réel", dont les trois récits déploient les diverses dissimulations à l'échelle planétaire, rappelle en effet une constellation littéraire plus ancienne: l'association secrète de comploteurs aux visées transnationales, dont les figurations se développent dans la fiction européenne, et en particulier française, au tournant du XIXe siècle. De Sand à Balzac en passant par Dumas ou Sue, des personnages conspirant au sein de mystérieuses sociétés prennent pour cible un personnage public, une institution, voire toute une société ou une population, à des fins de domination qui dépassent en l'occurrence le seul contexte français.

Que sa volonté de domination soit associée à une axiologie positive ou négative, tout comploteur développe envers sa cible des stratégies ressortissant au mensonge - que l'on associera au "plus petit dénominateur commun de toutes [s]es définitions" ${ }^{5}$ la dissimulation, qui procède d'une "divergence entre un sentiment ou une opinion d'une part et une énonciation ou une expression d'autre part", et "sert à des objectifs qui

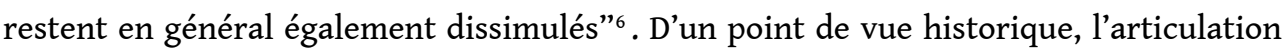
entre société secrète et mensonge à grande échelle est fondatrice d'un imaginaire dont 
les résurgences accompagnent chaque période de crise politique depuis environ deux

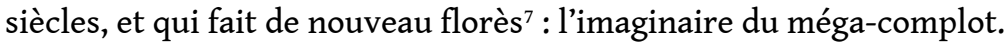

Quelques précisions s'imposent. Tout d'abord, il est ici question d'imaginaire social, au sens où l'entend notamment Dominique Kalifa : un "système cohérent, dynamique, de représentations du monde social, une sorte de répertoire des figures et des identités collectives dont se dote chaque société à des moments donnés de son histoire" ${ }^{\text {. }}$. En précisant qu'il "est surtout, comme le suggère Pierre Popovic, un ensemble interactif de représentations corrélées, organisées en fictions latentes", Kalifa souligne par ailleurs le rôle fondamental de la fiction dans la construction de l'imaginaire social - rejoignant par là un analyste plus direct des discours complotistes, Raoul Girardet, qui préfère, pour sa part, la notion de "mythe (politique)"10 à celle d'imaginaire. Chez les deux auteurs, le développement des discours et représentations étudiés est en outre associé à une période historique précise : la notion d'imaginaire - que nous emploierons sans adjectif - est donc résolument située. Pour le sujet qui nous intéresse, cette période débute au tournant du XIX siècle. Il a certes toujours existé des discours et fictions complotistes, mais le développement massif de récits mettant en scène des complots à visée transnationale débute à l'orée de l'ère contemporaine ${ }^{11}$. Dans ce contexte, la visée transnationale des complots se manifeste surtout par l'ambition dominatrice des figures de comploteurs, plus que par leur diversité nationale et/ou culturelle; l'une va souvent de pair avec l'autre, comme chez Bello, mais cette dernière n'est pas une nécessité ${ }^{12}$.

$4 \mathrm{Au} v \mathrm{vu}$ des caractéristiques historiques de l'imaginaire du méga-complot, dans quelle mesure les figures du mensonge relèvent-elles d'une représentation novatrice dans Les falsificateurs, Les éclaireurs et Les producteurs? Pour répondre à cette question, nous proposons de lire la trilogie de Bello comme la variation contemporaine d'un imaginaire complotiste transséculaire. Si les falsificateurs qui peuplent la série romanesque renvoient à certains traits structurels et narratifs récurrents dans les littératures en langues européennes depuis le tournant du XIX ${ }^{e}$ siècle, ces menteurs professionnels n'en dessinent pas moins une rationalisation de l'imaginaire du complot plutôt originale dans la littérature française. On pourrait dès lors se demander si Bello n'élaborerait pas des figures de menteurs post-romantiques, dans tous les sens du terme.

\section{Des menteurs qui en rappellent d'autres : retour(s) vers le passé}

5 Les trois romans de Bello font intervenir toute une galerie de fieffés menteurs. Le "Consortium de Falsification du Réel" (CFR), dont on apprend l'existence dans le 1er tome - situé au début des années 1990 - est une "organisation internationale occulte [...] qui opère sur les cinq continents et dans plus d'une centaine de pays", et dont les activités consistent à "modifie[r] la réalité" ( $F a 44-45)$. Cette falsification est surtout de deux ordres dans la trilogie, où elle peut consister :

- à altérer certains discours ou récits officiels associés à la vision des vainqueurs (ex. : truquer le manuscrit posthume d'un célèbre anthropologue pour sensibiliser à la cause des Bochimans du Kalahari, peuple d'Afrique australe menacé par l'exploitation industrielle des mines de diamant - première falsification mise en œuvre par le protagoniste sliv Dartunghuver, dans Les falsificateurs) et/ou allant à l'encontre d'une vision de l'Histoire fondée sur un idéal de concorde (ex. : donner de l'importance à Al Qaïda afin d'alerter les États-Unis sur la dangerosité de l'islamisme et éviter une escalade de violence - ce qui 
échoue lamentablement dans le $2^{\mathrm{e}}$ tome de la trilogie, qui gravite autour des attentats du $11 / 09)$;

- à inventer totalement un nouveau récit au service dudit idéal de concorde (ex. : créer une civilisation maya oubliée, les Chupacs - qui, contrairement aux autres Mayas, se serait caractérisée par sa non-violence, sa tolérance et son syncrétisme, dans le $3^{\mathrm{e}}$ tome de la trilogie).

Dans ce contexte, la falsification n'est possible que parce qu'elle est réalisée de manière dissimulée par les agents travaillant pour le CFR. Secret et mensonge sont d'autant moins dissociables dans la trilogie que les nouvelles recrues du CFR se demandent si l'Organisation ne leur dissimule pas des desseins néfastes (t. 1-2); et dans le t. 3, le Consortium est victime de falsificateurs qui minent son propre projet de falsification. Dans l'ensemble, les falsifications pratiquées en secret par les membres du CFR ont pour cible une opinion publique plus ou moins diffuse selon les projets dépeints, mais toujours transnationale, et qu'il s'agit de manipuler "à des fins [...] humanistes" (Pro 92).

On pourrait dès lors s'étonner qu'Antoine Bello, dans les commentaires et entretiens qu'il consacre à sa trilogie, ne mette pas en avant l'imaginaire (méga-)complotiste qui irrigue ses trois romans. "Pour moi, il s'agit avant tout d'un roman initiatique. Sliv, le personnage principal, cherche un sens à sa vie professionnelle", déclare-t-il au moment de la parution des Falsificateurs ${ }^{13}$, avant de renchérir, quelques années plus tard: "Je n'ai pas l'impression d'avoir fait un livre sur le complot. C'est avant tout l'histoire initiatique d'un jeune homme qui se cherche dans la vie, et qui va être confronté à la plus grande liberté qui soit: écrire l'Histoire"14. Il n'empêche que le protagoniste de Bello va trouver sa voie en lien avec la société secrète qui l'a recruté, dans une sorte d'écho au Wilhelm Meister de Goethe. Les années d'apprentissage de Wilhelm Meister (1795-1796) a en effet pour spécificité d'articuler le parcours d'un jeune homme au tempérament ludique avec les manigances d'une société secrète paramaçonnique ${ }^{15}$, qui suit ses succès et (surtout) ses déboires de façon plus ou moins distante, jusqu'à une fin heureuse signant pour le héros l'acceptation de soi et de sa place dans la société. Hormis le fait que le protagoniste de Bello sait dès le début qu'il évolue au sein d'une société secrète (dont il se détache ponctuellement au fil de la trilogie), ces caractéristiques de la célèbre œuvre de Goethe s'appliquent tout à fait aux trois romans de Bello. Sa trilogie pourrait ainsi être considérée comme une lointaine réécriture du modèle goethéen du Bildungsroman européen, qui a pour spécificité d'être aussi un Geheimbundroman, "roman de la société secrète"16.

7 Sans (encore) accuser Antoine Bello de mensonge, on peut dire que sa trilogie est par ailleurs truffée de traits structurels et narratifs typiques de la fiction du méga-complot, qui permettent de discerner, sous les personnages de falsificateurs œuvrant pour le CFR, un palimpseste plus diversifié que celui des Années d'apprentissage. Nous n'en mentionnerons que deux, en montrant leur ancienneté.

Première grande caractéristique de la fiction du méga-complot en langues européennes, la structure axiologique des trois romans est assez évidente. Bello construit une opposition d'ordre éthique entre le Consortium de Falsification du Réel et sa cible : la réalité. Si le CFR incarne un groupe de dissidents s'opposant aux institutions en place, leurs visées sont constructives: "Les sages de Toronto [qui fixent les orientations générales du CFR] [...] prêtaient le jour de leur nomination le serment de servir le genre humain en faisant abstraction des préjugés et des croyances qui avaient 
de tout temps divisé les peuples" ( $F a 469$ ). Les comploteurs éclairés de la trilogie de Bello visent ainsi à améliorer ce qui fait défaut dans la réalité socio-politique, chargée d'une axiologie négative. Cette définition s'appliquait déjà aux Invisibles et aux Illuminés peuplant respectivement La comtesse de Rudolstadt (1843) et Joseph Balsamo (1846-1849), comploteurs engagés contre les monarchies européennes. Elle n'empêche pas, que ce soit chez Sand, Dumas ou Bello, un floutage ponctuel de l'axiologie, visant à maintenir une tension narrative indispensable à des œuvres dépassant amplement les mille pages.

Deuxième grand trait de la fiction du méga-complot dont relève la trilogie de Bello, la dynamique de dévoilement qui l'anime renvoie à toute une tradition littéraire. Dans un mouvement paradoxal qui consiste à maintenir une forme de vérité au sein de la dissimulation, un enjeu essentiel du roman de Bello est de lever le voile sur la véritable finalité du CFR. À cet égard, des relances permanentes permettent, là encore, de nourrir la tension narrative jusqu'à la démystification. Dans Les falsificateurs, ce sont surtout les questions concernant la véritable finalité du CFR qui entretiennent cette tension. Elles débutent suite aux observations mystérieuses de l'sslandais Gunnar Eriksson, ayant tout juste proposé à Sliv Dartunghuver de travailler pour le Consortium :

je ne suis pas autorisé à vous en révéler bien davantage aujourd'hui. Je ne vous dirai pas par exemple qui dirige le CFR ni quand celui-ci a été créé.[...] À ma connaissance, le CFR ne poursuit pas de profit économique, de même qu'il ne cherche pas à prendre le pouvoir ou à faire accéder l'humanité à la sérénité cosmique. Les motifs du CFR, si motif il y a, sont d'une toute autre nature. (Fa 46-48)

Quelques pages plus loin, le personnage de Sliv inaugure la série de questions qui ponctue tout le roman :

Pourquoi le CFR falsifiait-il le réel? Avec quel argent et pour le compte de qui? Autant de questions auxquelles je n'avais toujours pas le moindre élément de réponse. Que ferais-je si j'apprenais d'ici à quelques semaines que l'organisation était financée par un gouvernement étranger à des fins subversives? ( $F a$ 73)

Ces questions seront ensuite relancées par Sliv lui-même, ainsi que par ses deux amis Magawati et Youssef, eux-mêmes agents, ou par d'autres membres du CFR (voir Fa 147, $317,370,392,422)$. Et elles reprendront dès le début du deuxième tome de la trilogie, où Sliv énonce: "Gunnar m'avait lancé un défi : Si l'on refuse de te révéler la finalité du CFR, pourquoi n'essaies-tu pas de la deviner ?" (Écl 20). Le véritable but du CFR sera dévoilé au terme de ce même volume : son absence de but précis - plus précisément, son pari sur l'efficacité de la concorde et du compromis. Là encore, rien de bien nouveau dans cette dynamique: autre exemple de roman mettant en scène une société secrète bénévolente, L'envers de l'histoire contemporaine de Balzac (1848) se caractérise par un procédé narratif assez semblable. Dans ce dernier volume de la Comédie humaine, le personnage de Godefroid ne rejoint les Frères de la Consolation que parce que sa curiosité l'a amené à questionner les visées de cette mystérieuse société catholique, qui lui seront dévoilées au cours du deuxième épisode du roman ("L'initié").

10 À l'instar d'autres romans récents, la trilogie de Bello rend ainsi caduque l'affirmation de Lyotard selon laquelle notre époque postmoderne signerait la "fin des grands récits" ${ }^{17}$. S'il est un grand récit qui a subsisté depuis son apparition au tournant du $\mathrm{XIX}^{\mathrm{e}}$ siècle dans les littératures en langues européennes, c'est bien celui du mégacomplot. Les menteurs-comploteurs de la trilogie de Bello sont les petits frères des Illuminés de Dumas, des Invisibles de Sand et des Frères de la Consolation de Balzac, à leur manière. Or, cette manière même est ce qui permet à Bello de créer une fiction 
plutôt originale au sein de la littérature française, notamment en se détachant du registre merveilleux qui émaillait les fictions complotistes de Dumas, Sand et, dans une certaine mesure, de Balzac.

\section{Des menteurs professionnels : une rationalisation de l'imaginaire du méga-complot}

11 En faisant se rencontrer un libéralisme bon teint, "à l'américaine", et un rationalisme optimiste "à la française" ${ }^{18}$, Antoine Bello réalise une variation assez inédite de l'imaginaire méga-complotiste associé à notre ère contemporaine. Ce choix est en partie lié au parcours de Bello, "l'un des rares écrivains français passés par le monde de l'entreprise, et même de la création d'entreprise"19 ainsi qu'à la posture assumée de "businessman dans la littérature" lui correspondant ${ }^{20}$. Au plan de la trilogie, le terme de rationalisation est sans doute le plus apte à déterminer la nature de cette variation.

12 Si l'on associe d'abord la rationalisation, au sens spécifique, économico-financier du terme, à un système de planification ayant pour but d'obtenir une meilleure productivité par l'organisation logique et méthodique, la trilogie peut être considérée comme une variation rationalisante de l'imaginaire du complot transnational. Le CFR possède en effet toutes les caractéristiques d'une multinationale: "Au-dessus des antennes se trouvent les bureaux, qui eux-mêmes rapportent aux centres" ( $F a 44)$, "le Plan est l'organe du CFR qui arrête les grandes orientations stratégiques de notre action" ( $F a 46)$, explique Gunnar Eriksson dès le début du roman. Et Sliv de renchérir, sur un ton plutôt critique, à la fin du 1er tome :

J'avais lu plusieurs ouvrages sur la théorie des organisations et, au vu de mon expérience, le CFR ne différait pas franchement d'entités plus traditionnelles, du type entreprises ou administrations. Les plus ambitieux, pour peu, naturellement, qu'ils possèdent les compétences nécessaires, prenaient inexorablement le pas sur les agents moins carriéristes, jusqu'à accaparer les postes à responsabilité et les rémunérations élevées. ( $\mathrm{Fa} 379$ )

Le lecteur est ici immergé dans le monde de l'entreprise, qui contraste avec la société illuminée à la Dumas ou avec le complot des bas-fonds à la Conrad ${ }^{21}$, pour ne citer qu'eux.

De ce point de vue, la fascination que nourrit Bello pour l'auteure nord-américaine Ayn Rand semble assez évidente. Dans son best-seller Atlas Shrugged (1957), encore assez peu connu du public français ${ }^{22}$, Rand reliait déjà l'imaginaire du méga-complot à l'univers des multinationales, dans une perspective pour le moins anti-socialiste ${ }^{23}$ : les conspirateurs qu'elle y met en scène sont des chefs d'entreprise incarnant la logique états-unienne du self-made man, et articulant une vision idéalisée du libéralisme économique à un froid pragmatisme. La veine épico-dystopique de ce roman à succès ne se retrouve toutefois pas vraiment dans la trilogie de Bello, qui insiste bien plus que Rand sur le quotidien de ses personnages. ${ }^{24}$

À cet égard, Bello ne se prive pas d'évoquer les aspects concrets du travail des agents, des lenteurs d'un agent parisien peu compétent, car proche de la retraite ( $F a$ 84), aux longs processus d'évaluation des dossiers produits par les falsificateurs, dont le quotidien n'est pas toujours palpitant : “J'avais l'impression de n'avoir jamais quitté les bancs de l'école; depuis maintenant dix ans, je rendais des copies" (Fa 433), observe 
ainsi Sliv. Les enjeux narratifs de cette dimension pragmatique semblent apparaître en filigrane dans les explications initiales de Gunnar Erikkson :

même quand [un jeune agent] accorde à la falsification l'importance qu'elle mérite, il se trahit par des procédés grossiers, empruntés aux mauvais romans d'espionnage, bien loin de ceux que nous employons quotidiennement sur le terrain. ( $F a 45)$

Si l'on ose en effet une interprétation méta-littéraire de ce passage, le transfert de l'imaginaire du méga-complot dans l'univers parfois terne de l'entreprise ressortirait ainsi à une volonté d'authenticité.

16 À un second niveau, le processus de rationalisation de l'imaginaire méga-complotiste à l'œuvre chez Bello s'explique par le fait que le projet et le fonctionnement du CFR sont fondés sur la raison. Le lecteur peut le discerner, dans une certaine mesure, dès la fin des Falsificateurs, où sont évoqués les "trois binômes de valeurs fondatrices" du CFR : "tolérance et relativité, liberté de corps et d'esprit, science et progrès" ( $F a 436)$. Mais il faut attendre le dénouement des Éclaireurs pour que soient révélées l'histoire et la finalité du Consortium. L'on découvre alors que les initiales "CFR" désignaient à l'origine une "Compagnie Française des Rentes", créée à la fin du xviIIe siècle par un "chevalier excentrique" prénommé Pierre Ménard - allusion transparente à Borges. Avant de mourir, le personnage avait ainsi engagé trois associés - un "rêveur exalté", un joueur de roulette et un "organisateur né" - sans leur expliquer en quoi consistait leur mission (Écl 366-374). Après avoir cherché, sans succès, les intentions de leur employeur, les trois hommes deviennent amis. Puis, en 1800, tous trois

renoncent solennellement à dissoudre l'organisation. Ils conservent les initiales CFR mais changent leur signification en Compagnie de Falsification de la Réalité. Ils entérinent également plusieurs principes [...] : la sécurité de la Compagnie prime sur toute autre considération; la direction est collégiale ; les effectifs refléteront la pluralité de la société. (Écl 375)

C'est afin de préserver cette pluralité que “[l]e CFR n'a pas de finalité" (Écl 376). L'équation rationnelle entre la diversité des opinions et l'absence de directive clivante fait écho à l'instigateur du CFR et à ses descendants éponymes ${ }^{25}$, dont le "sidérant éclectisme" va de pair avec l'esprit rationaliste des Lumières: on apprend que "[p]hilosophiquement parlant, il [Pierre Ménard] adhérait aux thèses d'un Voltaire ou d'un Montesquieu" (Écl 374), et qu'il serait entré au service de Diderot pour l'aider une fois que D'Alembert s'était retiré du projet de l'Encyclopédie (Écl 388).

17 Par la rationalisation qui la caractérise à différents niveaux, la trilogie de Bello manifeste ainsi sa spécificité par rapport au contexte éditorial français des dernières décennies. Les trois romans se distinguent notamment de l'imaginaire complotiste dont relève un autre roman à succès ancré dans le monde de l'entreprise : L'imprécateur (1974) de René-Victor Pilhes, où les sèmes du mystère et de l'obscurité sont bien plus présents $^{26}$. De même, Les falsificateurs, Les éclaireurs et Les producteurs de Bello ne s'inscrivent pas vraiment dans l'attrait renouvelé pour le (para-)religieux ${ }^{27}$ qui définit les fictions du méga-complot récentes parmi les plus lues en France, les best-sellers internationaux de Dan Brown en tête ${ }^{28}$. Cela n'empêche pas qu'une certaine foi se dégage de la trilogie, et oriente les falsifications qui y sont dépeintes : une foi placée dans les pouvoirs de la fiction. Celle-ci n'en est pas moins située du côté du mensonge un mensonge s'avérant néanmoins, dans les trois romans, bien plus noble que d'autres. 


\section{Des menteurs post-romantiques}

18 Si l'on songe aux deux sens usuels du terme "post-romantisme", les falsificateurs qui peuplent la trilogie de Bello semblent être de véritables produits de l'époque romantique, tout en possédant des traits postérieurs à cette époque ${ }^{29}$. C'est dans ce contexte que leur rapport à la fiction est essentiel. Les mensonges qu'ils créent peuvent d'abord être associés à un idéal-type romantique de la fiction, pour deux raisons principales: la conception totalisante de l'imagination qu'ils manifestent, mais aussi leur articulation à une force capable d'influencer l'Histoire humaine. Les personnages de menteurs-créateurs dont Bello nous dépeint les activités démiurgiques n'en sont pas moins ancrés dans notre époque actuelle, rendant compte d'un dépassement de l'épistémè romantique à laquelle ils ne se rattachent somme toute qu'en partie.

Avant de le montrer, il nous faut expliquer en quoi les agents du CFR, "maitres dans l'art de la falsification" ( $F a 40$ ), peuvent être rapprochés d'auteurs de fictions. Leur tâche consiste à rédiger des dossiers composés de deux parties: "Traditionnellement, l'auteur expose son scénario dans la première partie : que s'agit-il de faire croire ? Dans la deuxième partie, il dresse la liste des mesures à prendre pour crédibiliser le scénario" ( $F a$ 51). Cette bipartition correspond aux deux grandes qualités exigées des agents du CFR, incarnées de façon exacerbée par les personnages de Sliv Dartunghuver et de la froide Lena Thorsen: l'imagination, et une rigueur mise au service de l'authenticité. Mutadis mutandis, ces deux qualités renvoient à la tâche du romancier telle que définie par un Joseph Conrad: "My task which I am trying to achieve is by the power of the written word, to make you hear, to make you feel, it is, before all, to make you see. That, and no more, and it is everything" ${ }^{30}$. Si Conrad a ici surtout en tête l'importance de la sensation, sa remarque n'en renvoie pas moins aux frictions entre imagination et réel dont procède la fiction - produit de l'imagination ne possédant pas de modèle complet dans la réalité, mais s'y référant tout de même de manière ponctuelle ou émiettée ${ }^{31}$.

Or, dans la trilogie, la sympathie de l'instance narrative va clairement au personnage de Sliv et à ses capacités d'invention - que Bello définit comme un "pouvoir démiurgique qui est celui des mots" 32 . Selon une conception éminemment romantique de l'acte de création, les fictions créées par le personnage et les agents qui lui ressemblent ont ainsi le pouvoir de rendre cohérente une réalité présentée comme complexe. De fait, les falsificateurs cherchent à réaliser la synthèse de connaissances qu'ils ne cessent de cultiver au fil de la trilogie (voir en particulier Fa 383). Cette volonté d'accroissement du savoir articulée à une visée totalisante renvoie bien à deux traits essentiels du romantisme européen, comme le souligne Pierre Brunel :

pour donner une image juste du Romantisme, il ne suffit pas de sortir du domaine français pour réinsérer nos Romantiques dans le domaine européen, il ne suffit pas non plus d'élargir l'enquête littéraire à la philosophie, à la musique et aux beauxarts. Il faut retrouver l'assise d'une volonté de savoir. L'école romantique d'Iéna, si décisive pour l'histoire du Romantisme, a cherché à s'emparer de toutes les sciences, notamment des sciences naturelles et de la médecine, pour aboutir à une science totale, à une philosophie de la nature et de l'être humain, et découvrir le grand secret. Il en reste quelque chose quand Hugo part à l'écoute de la bouche d'ombre ou quand Gérard de Nerval veut retrouver les "Vers dorés" de Pythagore. ${ }^{33}$

La littérature et l'imagination jouent à ce titre un rôle privilégié, comme en témoignent la notion schlégélienne de "poésie universelle" ou le roman Henri d'ofterdingen de 
Novalis, qui répondent à la même ambition totalisante - résumée dans une célèbre formule de Jean Paul Richter : "L'imagination rassemble les éléments épars en un tout [...] et fait de toutes les parties du monde des mondes, elle totalise tout, même le Tout infini" ${ }^{34}$. En ce sens, les menteurs de Bello, comploteurs autant qu'écrivains - renvoyant par là au double sens du terme anglais plot, qui signifie "complot" mais aussi "fiction, intrigue" - peuvent être considérés comme des héritiers de l'époque romantique.

21 Nul hasard que la figure borgésienne de Pierre Ménard soit à l'origine du CFR : dans la célèbre nouvelle de l'écrivain argentin ${ }^{35}$, cette figure se caractérise non seulement par son éclectisme ${ }^{36}$, dont se ressaisit Bello, mais également par sa recherche d'une cohérence qui préside à sa réécriture de Don Quichotte. Cette recherche de cohérence est en l'occurrence toute personnelle: "Être, en quelque sorte, Cervantès et arriver au Quichotte lui sembla moins ardu - par conséquent moins intéressant - que continuer à être Pierre Mesnard et arriver au Quichotte à travers les expériences de Pierre Mesnard" ${ }^{37}$. C'est peut-être à la lumière de cet intertexte que l'on peut appréhender le mensonge qui conclut le $3^{\text {e }}$ tome de la trilogie de Bello, dans la section intitulée "Postface à la $23^{e}$ édition des Producteurs (juin 2026)" :

On a longtemps su peu de choses sur les circonstances dans lesquelles Anne-Lise Thorman a écrit la trilogie des Falsificateurs, publiée entre 2007 et 2015 dans une relative indifférence. Même si certains lecteurs avaient noté que les prénoms Lena et Nina forment un anagramme d'Anna-Line ou que Thorman résulte de l'agrégation de Thorsen (le patronyme de Lena) et de Schoeman (celui de Nina), ils en avaient conclu que l'auteur n'avait pu résister à la tentation qui frappe tant de jeunes auteurs de s'incarner dans leurs personnages. Il a fallu une interview donnée l'an dernier à l'occasion de sa nomination au poste de secrétaire générale des Nations Unies pour faire la lumière sur le caractère profondément autobiographique de ces trois romans. (Pro 567)

Ce dernier mensonge est celui de l'auteur même, nous proposant une mystification qui n'est certes pas des plus originales. Mais le but du passage est aussi d'associer la trilogie, et donc la fiction, à la production d'une cohérence individuelle : l'auteure fictive affirme en effet "s'être forgé les convictions qui ont guidé son parcours" grâce à l'écriture, et a ensuite "plac[é] son mandat [aux Nations Unies] sous le signe de la compréhension de l'autre" (Pro 571-572). Et le facétieux Bello de conclure : "Rien ne résiste à la littérature" (Pro 572), insistant de nouveau sur le pouvoir démiurgique des personnages d'écrivains qu'il met en scène au fil de ses trois romans.

Autre caractéristique mettant en évidence une continuité transséculaire, l'idée que les fictions élaborées par les falsificateurs sont capables d'influencer l'avenir est une constante dans la trilogie. Elle est assumée par le personnage de Sliv dans le passage suivant :

Même si j'avais l'impression de reconnaître mieux que la plupart de mes condisciples la mélodie enfouie dans la cacophonie de l'histoire, je n'avais jamais imaginé pouvoir contribuer à en écrire la partition. [...] Les analystes du Plan tâchaient de prédire plusieurs années à l'avance le moment où ces régimes qu'on aurait crus indestructibles s'écrouleraient brutalement sous le poids de leur incohérence. Les directives qu'ils rédigeaient ensuite ne visaient à rien de moins que précipiter l'histoire ( $F a$ 472-473)

Les complots du CFR apparaissent ici comme une formule miraculeuse permettant de modeler l'histoire sociale et politique. De ce point de vue, la rationalisation de l'imaginaire du méga-complot caractéristique de la trilogie ne dissimule pas entièrement sa dimension "magique". Là non plus, le romantisme n'est pas loin, comme le souligne Terry Eagleton, qui observe au sujet de cette période: "Literature has 
become a whole alternative ideology, and the imagination itself, as with Blake and Shelley, becomes a political force" ${ }^{38}$. On pensera aussi à Raoul Girardet, qui note qu' "[à] l'arrière-plan de la mythologie du Complot se découvre toujours, en fin de compte, la vision d'un Ordre, au sens religieux du terme, unitaire, conquérant, instrument nécessaire à la réussite d'une grande entreprise engageant et transcendant le destin de chacun" 39 - surtout pendant cet "Âge d'or de la Conjuration" qu'est le XIX ${ }^{\mathrm{e}}$ siècle européen ${ }^{40}$.

Une nouveauté dans la trilogie de Bello semble toutefois encourager son inscription dans un post-romantisme entendu cette fois comme un dépassement de la période romantique : la problématisation de la frontière entre mensonge et vérité. Celle-ci est notamment développée dans le passage suivant :

Les théories du complot fleurissaient dans les forums, recueillant un succès inversement proportionnel à leur plausibilité. Des sites en recensaient des pages entières, parmi lesquelles le visiteur pouvait choisir celles qui confortaient ses préjugés ethniques, politiques ou religieux : le FBI avait orchestré les attaques du 11 septembre, un petit nombre de patrons présidait aux destinées du monde, le gouvernement américain avait favorisé la propagation du sida au sein de la communauté noire, etc. Ces sornettes ne dataient pas d'hier mais Internet avait à la fois accéléré leur propagation et assis leur légitimité. Le concept de vérité n'avait jamais semblé si relatif. La Toile fournissait des arguments aux champions de toutes les causes, aux sionistes comme à ceux qui cherchaient des raisons de casser du Juif, aux tenants de l'évolution comme à ceux du créationnisme. Tout était vrai et donc rien n'était vrai ; tout était faux et donc rien n'était faux. (Pro 36-37)

Si la mise en question de la frontière entre mensonge et vérité ne date pas du tournant $\mathrm{du} \mathrm{XXI}{ }^{e}$ siècle $^{41}$, on peut néanmoins la considérer comme un trait massif de notre époque - en tout cas à l'échelle occidentale, comme le souligne entre autres Eva Illouz. Notant que "notre époque est marquée par le recul sans précédent d'un des principaux héritages des Lumières - la vérité en tant que pilier moral et politique"42, la sociologue renvoie à la distinction entre mensonge et bullshit dont traitent les travaux du philosophe Harry Frankfurt :

Le menteur ment parce qu'il tient à cacher la vérité; le diseur de conneries [bullshit], lui, ne poursuit même pas cet objectif. La vérité ne l'intéresse pas, ce qui l'intéresse, c'est que ses propos - vrais ou mensongers - aient un effet sur son auditoire, qu'ils lui permettent de renforcer son statut ou d'affaiblir son ennemi. ${ }^{43}$

Est-ce à dire que c'est finalement en s'intéressant au bullshit plus qu'au mensonge que Bello est le plus actuel ? Pas uniquement. Car c'est sur des "mensonges véritables", sur des dissimulations assumées, qu'insiste surtout l'écrivain dans les Producteurs, comme il le souligne dans un entretien accordé suite à la parution du roman :

L'élection de Bush et la guerre en Irak sont deux des plus grandes falsifications de l'Histoire. Mais dans ce livre, je veux aussi montrer la responsabilité écrasante du peuple américain : l'administration Bush a certes menti, mais on pensait que cette Constitution ne permettait pas ce mensonge, grâce au Congrès, à l'impeachment, ou au quatrième pouvoir (ndlr, les médias) ... Au bout du compte, il y a des gens qui mentent, et des gens qui acceptent qu'on leur mente. ${ }^{44}$

Dès lors, on visualise mieux le terrain glissant sur lequel s'aventurent de plus en plus certains personnages au fil de la trilogie. Dans la mesure où les falsifications sont omniprésentes dans nos sociétés, une entreprise de falsification s'inscrivant dans une visée immédiatement bénévolente - en l'occurrence, la concorde, et non l'encouragement ponctuel au conflit qui s'avère désastreux dans le $2^{\mathrm{e}}$ tome - devient éthiquement viable. C'est en tout cas ainsi que l'on peut interpréter la disparition des scrupules antérieurs 
de Sliv au terme du $3^{\mathrm{e}}$ tome du roman, qui se focalise sur l'invention d'une ancienne civilisation maya pacifique - mensonge créé de toutes pièces, mais mensonge visant la concorde, et donc "sacré cadeau [fait] à l'humanité" (Pro 373). Vive les fake news, tant qu'elles visent la paix et l'harmonie entre les peuples ! mensonge qui peuplent la trilogie de Bello sont essentiellement liées à son accueil, pas toujours très critique, de représentations et discours qui ont été d'abord importants dans le contexte nord-américain - que l'on pense à la fascination de l'écrivain pour l'univers d'Ayn Rand ou au rapport délicat à la vérité de ses personnages, qui rappelleraient parfois un Donald Trump, la bêtise et l'intolérance en moins. Les trois romans n'en ont pas moins l'intérêt d'incarner la figure du menteur dans celle du comploteur, et de souligner à ce titre la vivacité transséculaire d'un imaginaire mégacomplotiste qui a encore de beaux jours devant lui. À l'heure actuelle, Antoine Bello est en tout cas loin d'être le seul à se saisir d'un tel imaginaire. Outre feu Umberto $E \mathrm{EO}^{45}$, on pensera à des auteurs tels que le romancier nord-américain Thomas Pynchon $^{46}$, le Colombien Juan Gabriel Vásquez ${ }^{47}$, le Congolais In Koli Jean Bofane $e^{48}$, ou, dans le contexte français, Chloé Delaume ${ }^{49}$. Rappelant celui de leurs prédécesseurs, le succès de ces auteur'e's au tournant du XXI ${ }^{e}$ siècle invite ainsi à une étude comparatiste de la fiction du méga-complot dans une perspective transatlantique plus large.

\section{NOTES DE FIN}

1. L'éd. suivante sera convoquée dans cet article: Antoine Bello, Les falsificateurs [2007], Paris, Gallimard, 2008, <Folio> ; dorénavant Fa.

2. L'éd. suivante sera convoquée dans cet article: Antoine Bello, Les éclaireurs [2008], Paris, Gallimard, 2016, <Folio> ; dorénavant Écl.

3. L'éd. suivante sera convoquée dans cet article: Antoine Bello, Les producteurs [2015], Paris, Gallimard, 2016, <Folio> ; dorénavant Pro.

4. L'écrivain est par exemple qualifié de "prodige de l'écurie Gallimard" dans un article de Philippe Coste, “Antoine Bello, au-dessus de tout soupçon”, L'Express.fr [en ligne], 01/03/2009, disponible sur <http://www.lexpress.fr/culture/livre/antoine-bello-au-dessus-de-toutsoupcon_815624.html> (consulté le 15 juillet 2020).

5. Jochen Mecke, "Esthétique du mensonge", Cahiers d'études germaniques, $\mathrm{n}^{\circ} 68$, "Quelques vérités à propos du mensonge", vol. 2, 2015, p. 76. Disponible en ligne: <https:// journals.openedition.org/ceg/1436> (consulté le 15 juillet 2020.)

6. Ibid.

7. Sur ce point, nous nous permettons de renvoyer à notre article "Figuration des sociétés secrètes et méga-complot, $\mathrm{XIX}^{\mathrm{e}}-\mathrm{XXI}^{\mathrm{e}}$ siècles : de quelques continuités transséculaires et transnationales", SERD.hypotheses.org [en ligne], dossier "L'imaginaire des sociétés secrètes", Nicolas Aude et Marie-Agathe Tilliette (dirs), à paraître en 2021.

8. Dominique Kalifa, Les bas-fonds. Histoire d'un imaginaire, Paris, Seuil, 2013, <L'univers historique>, p. 20. 
9. Ibid., p. 21. - L'ouvrage auquel se réfère D. Kalifa est le suivant : Pierre Popovic, Imaginaire social et folie littéraire. Le Second Empire de Paulin Gagne, Montréal, Presses de l'université de Montréal, 2008, p. 24.

10. Voir Raoul Girardet, Mythes et mythologies politiques [1986], Paris, Seuil, 1990, <Points>, et en particulier la $1^{\text {re }}$ partie, «La conspiration », p. 25-62.

11. Sur le lien entre la Révolution française et l'émergence des discours (méga-)complotistes contemporains, voir notamment Aurélie Ledoux, Frédérique Leichter-Flack et Philippe Zard (dirs), Raison publique, n¹6, "Complot et terreur", Rennes, Presses universitaires de Rennes, 2012 ; Emmanuel Kreis, Les puissances de l'ombre. Juifs, jésuites, francs-maçons, réactionnaires... la théorie du complot dans les textes [2009], Paris, CNRS Éditions, 2012 ; Pierre-André Taguieff, L'imaginaire du complot mondial. Aspects d'un mythe moderne, Paris, Mille et une nuits, 2006. Dans une autre perspective, Maxime Decout identifie lui aussi la charnière entre le $\mathrm{XIX}^{\mathrm{e}}$ et le $\mathrm{XX}^{\mathrm{e}}$ siècle comme le moment où se développent le plus massivement des récits imposteurs (Pouvoirs de l'imposture, Paris, Minuit, 2018, <Paradoxe>).

12. De petits groupes de comploteurs peuvent chercher à mettre en place un complot mondial voire un "complot cosmique" - selon une formule d'Alessandro Leiduan dans Umberto Eco et les théories du complot. Contre le complotisme. Au-delà de l'anticomplotisme, Nice, Ovadia, 2019, $<$ Chemins de pensée>, p. 72 et al.

13. Antoine Bello, "Les falsificateurs. Présentation de l'auteur", Antoinebello.com [en ligne], 2007, disponible sur <https://www.antoinebello.com/les-falsificateurs> (consulté le 15 juillet 2020).

14. Thomas Mahler, "On flatte les gens dans leurs croyances", entretien avec Antoine Bello, Le Point, décembre 2011, reproduit sur le site d'Antoine Bello: <https://www.antoinebello.com/ presse> (consulté le 15 juillet 2020).

15. Voir en particulier Johnann Wolfgang von Goethe, Les années d'apprentissage de Wilhelm Meister [Wilhelm Meisters Lehrjahre, 1795-1796], trad. Blaise Briod [1954], éd. et trad. revue Bernard Lortholary [1999], Paris, Gallimard, 2007, <Folio classique>, livre VII, chap. IX, p. 602-609.

16. Voir à ce sujet Michael Voges, Aufklärung und Geheimnis. Untersuchungen zur Vermittlung von Literatur- und Sozialgeschichte am Beispiel der Aneignung des Geheimbundmaterials im Roman des späten 18. Jahrhunderts [1987], Berlin, de Gruyter, 2013, <Hermaea, Neue Folge>.

17. Jean-François Lyotard, La condition postmoderne, Paris, Minuit, 1979, p. 7 et al. - Nous renvoyons dans ce contexte à Jean-Pierre Dozon, "La fin des grands récits: un diagnostic occidentalo-centré", dans Gwenaëlle Lieppe, Laurent Lévi-Straus et Michel Wieviorka, Laurent (dirs), Penser global. Internationalisation et globalisation des sciences humaines et sociales, Paris, Éditions de la Maison des sciences de l'homme, 2015, p. 259-269, disponible en ligne : <http:// books.openedition.org/editionsmsh/4708> (consulté le 15/07/2020).

18. Nous nous inspirons ici d'une formule de Ludovic Barbiéri, qui souligne, dans une section d'article intitulée "Un libéral bon teint" : "Les producteurs illustre [...] la passion de l'organisation de Bello [...] qui trahit le businessman qu'il a été [...]. De même, tout dans Les producteurs témoigne de son tropisme américain : son état d'esprit, ses valeurs, sa façon de voir le monde sont imprégnés de libéralisme à l'ancienne, mélange d'optimisme, d'humanisme, de foi dans les capacités des hommes et des peuples à déplacer les montagnes", Ludovic Barbiéri, "Nouvelles leçons de falsification", Chronicart [en ligne], 20/03/2015, disponible sur <http:// www.chronicart.com/livres/nouvelles-lecons-de-falsifications/> (consulté le 15 juillet 2020).

19. Ibid.

20. Nous reprenons ici le titre d'un article de Michel Paquot, "Antoine Bello, un businessman dans la littérature" (Trends, août 2009), consultable dans la section "Presse" du site d'Antoine Bello comportant d'autres propos similaires : <https://www.antoinebello.com/presse> (consulté le 15 juillet 2020).

21. Voir en particulier Joseph Conrad, L'agent secret [The Secret Agent, 1907], trad. Sylvère Monod, Paris, Gallimard, 1995, <Folio classique>. Le roman met en scène un complot orchestré par une 
ambassade que l'on soupçonne russe, qui cible les milieux anarchistes londoniens tout en les manipulant partiellement.

22. Voir Ayn Rand, La grève [Atlas Shrugged, 1957], trad. Sophie Bastide-Foltz [2013], Paris, Les Belles Lettres, 2017 - édition où Antoine Bello est d'ailleurs remercié “pour [ses] encouragements" par la traductrice (p. 2).

23. Dans le roman, la "grève" du titre français vise à empêcher que le travail des brillants protagonistes, tous à la tête d'entreprises de poids, ne bénéficie aux tenants d'une économie plus solidaire.

24. De ce point de vue, l'univers terne que met en scène la trilogie de Bello se rapprocherait sans doute davantage de celui qui caractérise une série télévisée qui n'existait pas encore au moment de la rédaction des romans : Le bureau des légendes, créée par Éric Rochant et diffusée depuis avril 2015. Décrivant sur un mode réaliste le travail d'agents de renseignement affiliés à la DGSE, cette série française actuellement la plus exportée dans le monde se caractérise toutefois par une tension narrative plus forte que dans les trois romans de Bello, où les passages consacrés à la présentation détaillée des dossiers des falsificateurs sont très étendus.

25. Dans une démultiplication très borgésienne, le Français a eu un fils, prénommé comme lui, et ainsi de suite jusqu'aux début des années 2000, où un énième Pierre Ménard siège dans le comité exécutif du CFR (Écl 366).

26. Le roman culmine en une scène de meurtre exutoire, qui se déroule dans un souterrain labyrinthique accessible par un caveau du cimetière du Père-Lachaise. Voir René-Victor Pilhes, L'imprécateur [1974], Paris, Le Livre de poche, 1978, chap. XXV, p. 369-407.

27. Une exception concerne peut-être le statut de l'islam dans Les éclaireurs, tome où l'on apprend qu'Al Qaïda aurait été initialement créée par le CFR pour obliger les États-Unis à moduler leur politique étrangère - le directeur exécutif du CFR étant d'avis que "[d]e toutes les religions, l'islam sembl[e] de loin le mieux placé pour endosser le rôle de nouvel ennemi de l'Occident" (Écl 69). Une page plus loin, un glissement se produit, de "l'islam" à "l'islamisme radical" (Écl 40); et au moment où, le 11 septembre 2001, la famille soudanaise de Youssef assiste, en compagnie de sa fiancée Magawati (deux agents du CFR amis de Sliv), à l'explosion des tours jumelles, la jeune femme s'exclame : "Regarde ton père jubiler! De fait, Abdul arborait un mince sourire de conspirateur. Il avait passé son bras autour des épaules de son interlocuteur, comme s'il était sur le point de lui révéler l'emplacement du trésor des Templiers." (Écl 59) Le palimpseste complotiste apparaît ici dans toute sa profondeur historique, le topos (hélas) actuel du comploteur islamiste se superposant au motif ancien du trésor caché des Templiers, associant l'imaginaire complotiste à une dimension religieuse qui déborde l'actualité géopolitique dont il est du reste question.

28. Nous pensons entre autres aux deux ouvrages suivants: Dan Brown, Anges et démons [Angels and Demons, 2000], trad. Daniel Roche, Paris, Jean-Claude Lattès, 2005 ; Dan Brown, Da Vinci code [The Da Vinci Code, 2003], trad. Daniel Roche, Paris, Jean-Claude Lattès, 2004.

29. Nous nous référons ici aux deux significations de la notion que présente Terry Eagleton (même s'il rejette la seconde): “[W]e ourselves are post-Romantics, in the sense of being products of that epoch rather than confidently posterior to it", Terry Eagleton, Literary Theory. An Introduction [1983], Oxford, Blackwell Publishing, 1996, p. 16.

30. Joseph Conrad, "Preface", The Nigger of the Narcissus, New York, Doublebay, Page and Company, 1897, disponible sur <https://www.gutenberg.org/files/17731/17731-h/17731-h.htm> (consulté le 15 juillet 2020).

31. Voir Françoise Lavocat, Fait et fiction. Pour une frontière, Paris, Seuil, 2016, <Poétique>, p. 12 et al.

32. Thomas Mahler, op. cit.

33. Pierre Brunel, "Introduction", in Pierre Brunel (dir.), Romantismes européens et romantisme français, Montpellier, Espaces, 2000, p. 24. 
34. Jean Paul, "Vorschule der Ästhetik", Werke, Munich, Carl Hanser Verlag, 1973, tome V, p. 47, notre traduction.

35. Jorges Luis Borges, "Pierre Ménard, auteur du Quichotte" ["Pierre Menard, autor del Quijote", 1939], dans Fictions [Ficciones, 1956], trad. Nestor Ibarra et Paul Verdevoye [1957], Paris, Gallimard, 1980, <Folio>, p. 63-74. Sur l'importance de la figure de Pierre Ménard dans le contexte intellectuel et littéraire français, voir Fabula LHT [en ligne], $\mathrm{n}^{\circ}$ 17, "Pierre Ménard, notre ami et ses confrères", Arnaud Welfringer (dir.), juillet 2016, disponible sur <https://www.fabula.org/ lht/17/> (consulté le 15 juillet 2020).

36. Jorges Luis Borges, op. cit., p. 64-66.

37. Ibid., p. 68.

38. Terry Eagleton, op. cit., p. 17.

39. Raoul Girardet, op. cit., p. 61.

40. Ibid., p. 59.

41. Dans le contexte des théories de la fiction, voir Françoise Lavocat, op. cit., p. 117-146 en particulier; en lien avec une réflexion largement diachronique sur la mauvaise foi du roman, voir Maxime Decout, En toute mauvaise foi. Sur un paradoxe littéraire, Paris, Minuit, 2015, <Paradoxe>.

42. Eva Illouz, "Ces mensonges qui nous gouvernent" [Ha'Aretz, 14 septembre 2019], trad. Raymond Clarinard et al., Courrier international, $n^{\circ} 1513,31$ octobre 2019 - 6 novembre 2019, p. 33.

43. Ibid., p. 33-34.

44. Julien Bisson, "Antoine Bello : un homme de chiffres et de lettres", entretien avec Antoine Bello, France-Amérique, mai 2009, reproduit sur le site d'Antoine Bello: <https:// www.antoinebello.com/presse> (consulté le 15 juillet 2020).

45. Outre Le pendule de Foucault [Il Pendolo di Foucault, 1988] et Le cimetière de Prague [Il Cimitero di Praga, 2010] on pensera au dernier roman d'Umberto Eco, Numéro zéro [Numero Zero, 2015], trad. Jean-Noël Schifano, Paris, Grasset, 2015 - dont la portée transnationale est toutefois moins nette que dans les romans précédents.

46. Pour un roman récent, voir Thomas Pynchon, Fonds perdus [Bleeding Edge, 2013], trad. Nicolas Richard [2014], Paris, Seuil, 2015, <Points $>$.

47. Voir Juan Gabriel Vásquez, Le corps des ruines [La Forma de las Ruinas, 2016], trad. Isabelle Gugnon, Paris, Seuil, 2017, <Cadre vert>.

48. Voir In Koli Jean Bofane, Mathématiques congolaises [2008], Arles, Actes Sud, 2011, <Babel>.

49. Voir Chloé Delaume, Les sorcières de la République, Paris, Seuil, 2016, <Fiction et $\mathrm{C}^{\mathrm{ie}>}$.

\section{RÉSUMÉS}

La trilogie Les falsificateurs (2007), Les éclaireurs (2009) et Les producteurs (2015) a valu à Antoine Bello un succès rapide. Si certains ont pu admirer sa popularité soudaine, force est de constater que les figures du mensonge qui peuplent sa trilogie ne sont pas nouvelles. Le "Consortium de Falsification du Réel", dont les trois romans déploient les multiples dissimulations, rappelle ainsi une constellation littéraire plus ancienne: l'association secrète de comploteurs aux visées transnationales. À ce titre, nous proposons de lire la trilogie de Bello comme la variation contemporaine d'un imaginaire transséculaire. Une mise en perspective historique révèle en effet un palimpseste complotiste sous les personnages de falsificateurs que l'écrivain met en scène. Par ailleurs, ces menteurs professionnels font émerger une dynamique plutôt originale dans la littérature française : une rationalisation de l'imaginaire du méga-complot, dont nous tenterons de cerner les modalités, les enjeux et les limites. 
INDEX

Mots-clés : falsification, dissimulation, fiction du complot, post-romantisme

AUTEUR

CHLOÉ CHAUDET

Université Clermont Auvergne 